\title{
Meralgia paresthetica following hemorrhoidectomy in the jack-knife position - A case report -
}

Received May 3, 2018

Revised 1st, July 31, 2018

2nd, September 17, 2018

Accepted September 17, 2018

\section{Corresponding author}

Hyung Rae Cho, M.D.

Department of Anesthesiology and

Pain Medicine, Myongii Hospital,

Hanyang University College of

Medicine, 55 Hwasu-ro 14beon-gil,

Deogyang-gu, Goyang 10475, Korea

Tel: 82-31-810-6200

Fax: 82-31-810-6203

E-mail: callmex@hanmail.net

ORCID

https://orcid.org/0000-0003-1634-7482

\section{Hyeon Jun Yang, Jun Sung Yoo, Jin A Kim, Yoo Kang, Yong Kyung Lee, Jin Hye Min, and Hyung Rae Cho}

Department of Anesthesiology and Pain Medicine, Myongji Hospital, Hanyang University College of Medicine, Goyang, Korea

Meralgia paresthetica (MP) is a neuropathic pain caused by the entrapment of the lateral femoral cutaneous nerve (LFCN). There have been reports of MP following various surgeries; however, it has not yet been reported after hemorrhoid surgery. We report a case of bilateral MP after hemorrhoid surgery in a jack-knife position. The patient presented with pain, tightness, and a tingling sensation in the anterolateral aspect of both thighs. Ultrasonography-guided LFCN block was used for diagnosis and treatment, along with conservative management for 20 days with oral medication. One month later, the patient's symptoms had resolved completely. MP due to the jack-knife position may occur postoperatively in patients with predisposing risk factors such as obesity and diabetes mellitus, despite adequate padding and a shorter operating time.

Keywords: Femoral neuropathy; Hemorrhoidectomy; Nerve compression syndrome; Neuralgia; Prone position.

Meralgia paresthetica (MP) is a neuropathic pain caused by entrapment of the lateral femoral cutaneous nerve (LFCN), leading to pain, tingling sensation, and numbness in the anterolateral aspect of the thigh. MP has an incidence rate of 4.3 per 10,000 person-years in the general populations. It has been reported to be associated with obesity, diabetes mellitus (DM), prolonged surgical time, and other entrapment etiologies including pregnancy, wearing tight clothes, and leaning against hard objects $[1,2]$. It is also suspected that these patients develop MP due to direct compression of the LFCN in the prone position. When a hemorrhoid surgery is conducted in the jack-knife position, the weight of the abdominal viscera and adipose tissue may be subject to greater traction forces, resulting in increased compression of the LFCN. MP may develop in severe obese patients even if with short surgical time.

To the best of our knowledge, this case report would be the first case of MP due to the jack knife position assumed in hemorrhoid surgery.

\section{CASE REPORT}

A 42-year-old man (height $176 \mathrm{~cm}$; weight $140 \mathrm{~kg}$; body mass index [BMI] $41.6 \mathrm{~kg} / \mathrm{m}^{2}$ ) diagnosed with hemorrhoids, underwent hemorrhoidectomy surgery. The patient had a history of hypertension, diabetes, heart failure, and restless leg syndrome (RLS). He was under treatment with antidepressants, including a selective serotonin receptor inhibitor (SSRI) and benzodiazepines (BZD), along with antihypertensive drugs. Initially, surgery under spinal anesthesia in a jack-knife position was planned. Considering the BMI of the patient, a blind saddle block was attempted; unfortunately, it failed. Following this, an ultrasonography-guided spinal

This is an Open Access article distributed under the terms of the Creative Commons Attribution Non-Commercial License (http://creativecommons.org/licenses/by-nc/4.0) which permits unrestricted non-commercial use, distribution, and reproduction in any medium, provided the original work is properly cited.

Copyright (c) the Korean Society of Anesthesiologists, 2019 
block was attempted; however, we realized that our needle was too short to penetrate the dura of the patient. Hence, general anesthesia was decided as the final resort, and was induced using $200 \mathrm{mg}$ of propofol, $100 \mu \mathrm{g}$ of fentanyl, and 80 $\mathrm{mg}$ of rocuronium. When muscle relaxation was achieved, the patient was intubated with a laryngeal mask airway (LMA) (I-gel, Intersurgical Ltd., UK). Anesthesia was maintained with $1 \mathrm{~L} / \mathrm{min}$ oxygen, $1 \mathrm{~L} / \mathrm{min}$ nitrous oxide, and 1.5 volume\% sevoflurane, with ventilator settings of $750 \mathrm{ml}$ tidal volume and a respiratory rate of 11 breaths/min. The concentration of sevoflurane was appropriately adjusted to maintain a bispectral index of 40-60. The surgery was conducted in the jack-knife position for a duration of 90 minutes. The head, face, shoulders, arms, hips, and feet of the patient were supported with soft foam or rolls to prevent bony pressure and other pressure injuries. The vital signs were stable and did not need adjustment. At the end of the surgery, when skin suturing was initiated, muscle relaxation reversal with $0.4 \mathrm{mg}$ of glycopyrrolate and $20 \mathrm{mg}$ of pyridostigmine was performed. After confirming that the patient's breathing was regained satisfactorily, the LMA was removed gently. The patient was observed in a postoperative anesthetic care unit for an additional 60 minutes and then transferred to the ward. On the second postoperative day, the patient complained of pain, tightness, and a tingling sensation in the anterolateral aspect of both thighs. The numeric rating scale (NRS) score (0: no pain; 10: worst imaginable pain) was about $7-8$ at most times, occasionally increasing to 10 at bedtime. As the patient was already consuming antidepressants, such as BZDs and SSRIs for RLS, we prescribed $225 \mathrm{mg} /$ day pregabalin, $10 \mathrm{mg}$ /day prednisolone, and $300 \mathrm{mg} /$ day tramadol. Three days later, the patient did not experience any change in symptoms, and we increased pregabalin dose from $225 \mathrm{mg}$ to $300 \mathrm{mg} /$ day. Two days after the change in dosage, the patient's discomfort was still not alleviated, and the pregabalin dose was increased to $450 \mathrm{mg} /$ day after confirming that the patient had no side effects with the previous doses. Despite an increase in pregabalin dose for seven days, the patient's symptoms did not improve. Since the initial treatment was not effective, we performed a LFCN block. Before the needle insertion, fascia lata, fascia iliaca LFCN, arteries, and veins were scanned along the inguinal line from the lateral aspect above the anterior superior iliac spine (ASIS) to the medial aspect using a high-frequency linear ultrasound transducer (8-15 MHz, L8-
15IS linear probe, Samsung Medison Co. Ltd., Korea). Following local anesthetic infiltration while avoiding the blood vessels, the needle (Uniplex Nanoline, 22-gauge $\times 100 \mathrm{~mm}$, PAJUNK $^{\circledast}$, Germany) was introduced in plane from lateral to medial. When the position of the needle tip was judged appropriate, $2 \mathrm{ml}$ of normal saline was injected. When it was confirmed that the patient did not have pain and the spread of the bolus was appropriate, a mixture of triamcinolone 20 $\mathrm{mg}+0.125 \%$ ropivacaine $10 \mathrm{ml}$ was injected. Additionally, we further increased the pregabalin dose to $600 \mathrm{mg} /$ day and prescribed $50 \mathrm{mg}$ of tapentadol, before bedtime, to prevent sudden episodes of pain in sleep. Ten days following this, the patient reported that there was almost no pain or tingling sensation during the day but that sudden symptoms only appeared at bedtime. Due to concerns of possible side effects, such as nerve injury or pain exacerbation, the patient refused further block administration. The worst NRS during sleep was 3 points. The patient reported that sleep was occasionally disturbed by pain but the pain was not severe. Fortunately, the symptoms improved dramatically and the dose of the drugs could be tapered. The patient was informed that the pain may recur, and was advised to take $50 \mathrm{mg}$ of tapentadol, $100 \mathrm{mg}$ of tramadol, and $150 \mathrm{mg}$ of pregabalin for three days, before bedtime. Three days later, the NRS was less than 2 at bedtime, and we decided to discharge the patient. One month after discharge, the patient reported complete resolution of symptoms.

\section{DISCUSSION}

Although there have been reports of occurrence of MP in surgical or non-surgical situations, there have been no reports of MP after hemorrhoidectomy. MP reportedly occurs most often between 30 and 40 years of age, and the incidence is 4.3 per 10,000 person years [1]. Until now, autosomal predominance of MP is unclear, although a case of family study including four generations was reported, and there is no consensus on sex predominance [3]. Generally, MP is unilateral but sometimes could be bilateral [4].

MP can be classified as spontaneous or iatrogenic, and may have more than 80 etiologies [5]. Irrespective of the cause, MP is known to be induced by mechanical compression of the LFCN, but sometimes it may be caused by underlying conditions such as DM, obesity, alcoholism, hypothyroidism [6], 
degenerative spinal disorders, and prolonged surgical time [2]. Additionally, a recent study claimed that each risk factor is not independent, but interlinked to induce MP [7]. The patient in this case had DM and high BMI for Korean standards, and these factors might have contributed to the development of MP.

MP has been reported to occur following surgeries such as laparoscopic surgery, coronary artery bypass grafting, aortic valve surgery, hip surgery, spine surgery, appendectomy, gynecologic surgery, and inguinal hernia repair [5]. Operative time longer than 3.5 hours, high BMI, inadequate surgical positioning, and iliac crest harvesting are risk factors for LFCN injury associated with surgery [2]. Some studies have reported that intra-operative hypotension and significant blood loss are possible risk factors $[2,8]$. Hemorrhoidectomy in this patient was performed in the jack-knife position, but padding was applied properly and the operation time did not exceed 2 hours. Additionally, there was no intra-operative hypotension or bleeding. Therefore, demographic factors of the patient including high BMI and DM themselves were inferred to have induced MP rather than the intra-operative factors.

It has been reported that MP did not worsen with positions such as standing or walking [9], as observed in this patient too. However, when the pressure was applied around the ASIS during sleep, the pain aggravated.

The LFCN, which is the nerve involved in MP, is primarily a sensory nerve originating from the first through the third lumbar nerve roots [4]. It can be localized passing over, under, or through the inguinal ligament. Depending on the pathway and the location in relation to the ASIS, two to five possible variations have been reported [10]. Due to variations, ultrasonography is technically feasible and is the accurate method for the diagnosis of MP [11]. For the treatment of MP, removal of the source of compression is of utmost importance, but conservative therapy can also be attempted with effective therapies (non-steroidal anti-inflammatory drugs, antidepressants, anticonvulsants, and physical therapy are known to be effective). Despite this initial treatment, if the patient's symptoms persist, injection of the LFCN can be attempted. LFCN blocking can be performed using a landmark-based or imaging-guided method [12]. In the landmark-based method, the needle is inserted $2.5 \mathrm{~cm}$ medial to the ASIS, just caudal to the inguinal ligament. The success rate of the landmarkbased method is lower than $40 \%$ [13], and is attributed to various anatomic variations of LFCN. On the other hand, the ultrasound-guided method with the aid of a peripheral nerve stimulator has a high success rate of about $85 \%$ [11]. Thus, for a high success rate of blockade, image-guided LFCN block is recommended. In this patient, the nerve-conduction velocity test was rejected. Therefore, LFCN block was performed for diagnosis and treatment under ultrasound guidance; the patient's LFCN was found to pass under the inguinal ligament and showed hypoechogenicity. There was pain relief following the LFCN block. When non-surgical treatment fails and symptoms become intractable, surgical treatment needs to be considered. In this case, surgical treatment was excluded because the patient's symptoms responded at least partially to the initial conservative treatment.

In conclusion, clinical physicians should remember that MP may occur in the jack-knife position of hemorrhoidectomies, despite short operating times and adequate padding. Patients with predisposing risk factors such as DM and obesity should be pre-informed of the possibility of occurrence of MP postoperatively.

\section{CONFLICTS OF INTEREST}

No potential conflict of interest relevant to this article was reported.

\section{ORCID}

Hyeon Jun Yang: https://orcid.org/0000-0003-1511-0168

Jun Sung Yoo: https://orcid.org/0000-0002-0946-4464

Jin A Kim: https://orcid.org/0000-0002-1711-7243

Yoo Kang: https://orcid.org/0000-0001-6330-8086

Yong Kyung Lee: https://orcid.org/0000-0002-8921-8174

Jin Hye Min: https://orcid.org/0000-0002-0259-6501

\section{REFERENCES}

1. van Slobbe AM, Bohnen AM, Bernsen RM, Koes BW, BiermaZeinstra SM. Incidence rates and determinants in meralgia paresthetica in general practice. J Neurol 2004; 251: 294-7.

2. Yang SH, Wu CC, Chen PQ. Postoperative meralgia paresthetica after posterior spine surgery: incidence, risk factors, and clinical outcomes. Spine (Phila Pa 1976) 2005; 30: E547-50.

3. Malin JP. Familial meralgia paresthetica with an autosomal 
dominant trait. J Neurol 1979; 221: 133-6.

4. Ivins GK. Meralgia paresthetica, the elusive diagnosis: clinical experience with 14 adult patients. Ann Surg 2000; 232: 281-6.

5. Harney D, Patijn J. Meralgia paresthetica: diagnosis and management strategies. Pain Med 2007; 8: 669-77.

6. Grossman MG, Ducey SA, Nadler SS, Levy AS. Meralgia paresthetica: diagnosis and treatment. J Am Acad Orthop Surg 2001; 9 : 336-44.

7. Weng WC, Wei YC, Huang WY, Chien YY, Peng TI, Wu CL. Risk factor analysis for meralgia paresthetica: a hospital-based study in Taiwan. J Clin Neurosci 2017; 43: 192-5.

8. Gupta A, Muzumdar D, Ramani PS. Meralgia paraesthetica following lumbar spine surgery: a study in 110 consecutive surgically treated cases. Neurol India 2004; 52: 64-6.

9. Parisi TJ, Mandrekar J, Dyck PJ, Klein CJ. Meralgia paresthetica: relation to obesity, advanced age, and diabetes mellitus. Neurology 2011; 77: 1538-42.

10. Üzel M, Akkin SM, Tanyeli E, Koebke J. Relationships of the lateral femoral cutaneous nerve to bony landmarks. Clin Orthop Relat Res 2011; 469: 2605-11.

11. Ng I, Vaghadia H, Choi PT, Helmy N. Ultrasound imaging accurately identifies the lateral femoral cutaneous nerve. Anesth Analg 2008; 107: 1070-4.

12. Özçakar L, Kara M, Yalçın B, Yalçın E, Tiftik T, Develi S, et al. Bypassing the challenges of lower-limb electromyography by using ultrasonography: AnatoMUS-II. J Rehabil Med 2013; 45: 604-5.

13. Shannon J, Lang SA, Yip RW, Gerard M. Lateral femoral cutaneous nerve block revisited. A nerve stimulator technique. Reg Anesth 1995; 20: 100-4. 